\title{
High-Precision Repetitive Motion Control in Permanent Magnet Synchronous Motor Using a Periodic Adaptive Extended State Observer
}

\author{
Zhi Ren ${ }^{1}$, Wenjun Qiao", Yang Liu', Xiaohong Zhang ${ }^{1}$, Songtao Li ${ }^{1}{ }^{\text {* }}$, Min Huang ${ }^{2}$, Yingdi Bao ${ }^{2}$, \\ Gang Fang ${ }^{2}$ \\ ${ }^{1}$ School of Mathematics and Physics, North China Electric Power University, Baoding, China \\ ${ }^{2}$ GoodWe (Jiangsu) Power Supply Technology Co. Ltd, Suzhou, China
}

Email address:

songtaoli2001@126.com (Songtao Li)

${ }^{*}$ Corresponding author

\section{To cite this article:}

Zhi Ren, Wenjun Qiao, Yang Liu, Xiaohong Zhang, Songtao Li, Min Huang, Yingdi Bao, Gang Fang. High-Precision Repetitive Motion Control in Permanent Magnet Synchronous Motor Using a Periodic Adaptive Extended State Observer. American Journal of Chemical Engineering. Vol. 4, No. 5, 2016, pp. 122-130. doi: 10.11648/j.ajche.20160405.15

Received: September 21, 2016; Accepted: October 14, 2016; Published: October 20, 2016

\begin{abstract}
This paper focuses on estimating and compensating the lumped disturbances in the repetitive motion control system of permanent magnet synchronous motor. A Periodic Adaptive Extended State Observer (PAESO) consisting of a normal Extended State Observer (ESO) and a refined ESO is proposed. The normal ESO is designed to capture the approximate range of the disturbances, and the refined ESO is developed to optimize the quantity of the estimated lumped disturbances by integrating real-time position tracking error. Based on PAESO, a hybrid controller, including a feedback branch and a feedforward branch, is presented to make the overall system stable and the position tracking error convergent towards zero. The effectiveness of the proposed PAESO is verified through three real-time experiments. Experimental results show that a high-precision tracking performance is obtained by the proposed PAESO.
\end{abstract}

Keywords: Permanent Magnet Synchronous Motors, Lumped Disturbances, Periodic Adaptive Extended State Observer, Repetitive Position Control

\section{Introduction}

Ever-increasing demands on advanced manufacturing applications, such as gantry robots [1], wafer scanners [2], have continued to motivate and stimulate the development of the high-speed and high-precision repetitive motion control system. Among the various electric motor drives, permanent-magnet synchronous motor (PMSM) is probably the most suitable choice for these situations because of its advantages such as high efficiency, fast dynamic and easy maintenance [3], [4]. However, in practice, PMSM systems always suffer from different disturbances, which may arise due to the load variations, the system parameters perturbations owing to prolonged usage and the inherent nonlinear dynamics. Specifically, two major nonlinear phenomena faced by PMSM are torque ripple and friction [5], [6]. Torque ripple is usually generated periodically by various sources, including cogging torque, flux harmonics, and current sensor errors, which deteriorates position tracking accuracy significantly [7]. Friction, another commonly encountered disturbance, causes the limit-cycle oscillation, small steady state error, and other undesirable influences [5].

At continuous motion systems, great deals of observer-based efforts have been made to overcome the adverse influences of disturbances. One popular and simple approach is the disturbance observer (DOB) [8]. Elfizy et al. [9] and Yan et al. [9] apply DOB to reduce the adverse effects caused by friction force, force ripple, and external load disturbances. In [10], a self-tuning DOB is adopted in speed control system to compensate the external disturbances. Huang et al. [11] design DOB in state space to estimate the parameter uncertainties and current measurement errors in the velocity and current loop of the servo driver, respectively. In 
order to depress the high frequency elements from the measurement noises or the unmodeled dynamics, a low-pass Q-filter is introduced to limit the bandwidth of DOB, which, however, may cause magnitude distortion as well as phase lag of the estimated disturbances [12], [13]. Another well-developed observer technique is extended state observer (ESO), which is originally presented by Han [14], [15]. The ESO treats the lumped disturbances, which consist of the internal uncertainties and the external disturbances, as a new state of system. In [16], a linear adaptive ESO is designed for the speed regulation control system of PMSM. Feng et al. [17] exploit ESO to accurately estimate the derivative signals in induction motors. In [18], Xu et al. employ ESO to estimate and compensate the unknown disturbances and embed the estimation result into a robust automatic disturbances rejection controller. Even though ESO is powerful and efficient in estimating the lumped disturbances, it takes time to asymptotically converge to the actual quantity of the lumped disturbances, and a small steady deviation always exists when the lumped disturbances are time-varying. Therefore, the characteristic of phase hysteresis in ESO is always unavoidable.

In a repetitive motion control system which performs a fixed task repeatedly, precise estimation and compensation of disturbances become possible owing to the fact that large parts of the disturbances in PMSM system have periodic and repetitive features [29]. Since the disturbances in repetitive process have the same time period with position reference, an effective approach catering to this problem is the learning strategy. Iterative learning control [20], [21], repetitive control [22], and periodic adaptive learning control [23]-[28] have been proposed to estimate and attenuate the periodic disturbances. In [20], the time-domain and frequency domain iterative learning control strategies are conducted respectively to reduce the periodic torque ripple. In [27], Ahn et al. compensate the cogging and coulomb friction by using information from last period to update current adaptive laws. Generally, in iterative learning control and repetitive control, an inverse model of the closed-loop transfer function should be utilized to design the learning filter and to guarantee the tracking error convergent. In addition, it is not easy to make these two controllers stable for all unknown disturbances. In periodic adaptive learning control, a nonlinear function is required to acquire the initial quantity of the lumped disturbances, which may be difficult and complicated to be designed [29]. In order to deal with this problem, a periodic adaptive disturbance observer [29], [30] is proposed where the classical linear DOB is used to design the initial conditions, and then the periodic adaptive law is adopted to update and compensate these disturbances. But the design of the cutoff frequency for Q-filter is of great importance in balancing between different factors, for example, stability and response requirements of the control system, frequency characteristic of the external disturbances and measurement noises [8].

Motivated by the above ideas, a periodic adaptive ESO (PAESO), composed of a normal ESO and a refined ESO, is proposed to estimate and attenuate the periodic disturbances on the repetitive motion control system of PMSM. There are four main contributions in the PAESO. Firstly, the disturbances are treated in a lumped form and estimated roughly by the normal ESO. Secondly, because of the characteristics of phase lag and amplitude distortion in the normal ESO, a refined ESO is employed to update the estimated quantity of the lumped disturbances and to make it converge to the actual disturbances gradually. Thirdly, based on PAESO, a hybrid controller made up of by a feedback branch and a feedforward branch is proposed to make the overall system stable and the position tracking error convergent asymptotically. Finally, real-time experiments are performed to verify the effectiveness of the proposed PAESO.

This paper is organized as follows. Section 2 presents the dynamic model of the repetitive motion control system of PMSM and the knowledge of ESO briefly. Some properties and notations about the repetitive process are also listed for convenience of description. Section 3 illustrates the design details of the hybrid controller at first. Then the stability of PAESO are discussed in details. Real-time experiments are conducted to verify the superiority of proposed PAESO in Section 4. Section 5 contains some conclusions.

\section{Background}

In this section, the dynamics of repetitive motion control system of PMSM are expressed at first. Then, a simplified model with lumped disturbances is presented. Next, the normal ESO is introduced to estimate the lumped disturbances. Some properties and notations in repetitive motion process are listed to facilitate the description.

\subsection{Model of PMSM}

Without loss of generality, the mechanical dynamics of PMSM systems under the vector control strategy are expressed as follows:

$$
\left\{\begin{array}{l}
\dot{\theta}(t)=\omega(t) \\
J \dot{\omega}(t)=T_{e}(t)-T_{\text {load }}(t)-T_{\text {fric }}(\theta, \omega, t)-T_{\text {rip }}(\theta, \omega, t)-T_{n}(\theta, \omega, t) \\
T_{e}(t)=K_{f} i_{q}(t)
\end{array}\right.
$$

where $\theta(t)$ and $\omega(t)$ are the motor angular position and speed respectively; $J$ is the angular momentum of inertia; $T_{e}(t)$ is the designed motor torque; $T_{\text {load }}(t), T_{\text {fric }}(\theta, \omega, t)$, $T_{\text {rip }}(\theta, \omega, t)$ and $T_{n}(\theta, \omega, t)$ are the disturbance torques derived from load, friction, ripple, and other unmodeled characteristics, respectively; $K_{f}$ is the constant torque coefficient; $i_{q}(t)$ is the $q$-axis torque current. Actually, the electrical dynamics are fast enough compared with the frequency bandwidth of interest and are ignored for simplicity.

These disturbances are rearranged in a unified form as follows:

$$
d(t)=T_{\text {load }}(t)+T_{\text {fric }}(\theta, \omega, t)+T_{\text {rip }}(\theta, \omega, t)+T_{n}(\theta, \omega, t)
$$


where $d(t)$ represents the sum of disturbances.

Using (1) and (2), the simplified controlled model is expressed below.

$$
\left\{\begin{array}{c}
\dot{\theta}(t)=\omega(t) \\
\dot{\omega}(t)=-a(t)+u(t)
\end{array}\right.
$$

where $u(t)=K_{f} i_{q}(t) / J ; a(t)=d(t) / J, a(t)$ represents the lumped disturbances.

\subsection{Extended State Observer}

It is well-known that, the ESO technique regards the lumped disturbances as a new state of system [14]. In (3), define $x_{1}=\theta(t), x_{2}=\omega(t)$, and $a(t)$ as an extended state $x_{3}=-a(t)$. Then the controlled model (3) is rewritten as:

$$
\left\{\begin{array}{l}
\dot{x}_{1}=x_{2} \\
\dot{x}_{2}=x_{3}+u(t) \\
x_{3}=-a(t)
\end{array}\right.
$$

According to (4), a nonlinear ESO is constructed as follows [14]:

$$
\left\{\begin{array}{l}
e=z_{1}-x_{1}, f e_{1}=f a l(e, 0.5, \delta), f e_{2}=f a l(e, 0.25, \delta) \\
\dot{z}_{1}=z_{2}-\beta_{1} e \\
\dot{z}_{2}=z_{3}-\beta_{2} f e_{1}+u \\
\dot{z}_{3}=-\beta_{3} f e_{2}
\end{array}\right.
$$

where $z_{1}, z_{2}$ and $z_{3}$ are the respective estimations of $x_{1}$, $x_{2}$ and $x_{3} ; e$ is the estimate error; $\beta_{1}, \beta_{2}$ and $\beta_{3}$ are the observer gains. $f a l(e, \alpha, \delta)$ is a nonlinear function and defined as [14]:

$$
f a l(\mathrm{e}, \alpha, \delta)= \begin{cases}\frac{e}{\delta^{1-\alpha}} & |e|<\delta \\ |e|^{\alpha} \operatorname{sign}(\mathrm{e}) & |\mathrm{e}|>\delta\end{cases}
$$

The reason that the $f a l(\mathrm{e}, \alpha, \delta)$ function has been chosen in (5) is that such a nonlinear function provides surprisingly better results in practice [14], [19]. Compared with the linear feedback, the estimate error $e$ approaches zero much more quickly in infinite time when the nonlinear feedback of the form $|e|^{\alpha} \operatorname{sign}(\mathrm{e})$ with $\alpha<1$ is employed. The nonlinearity of $f a l(\mathrm{e}, \alpha, \delta)$ is efficient for reducing steady state error, which avoids an integral control. More details about ESO can be found in [14].

When the estimate error $e$ comes closely to zero, the estimate states $z_{1}, z_{2}$ and $z_{3}$ will be approximately equal to $x_{1}, x_{2}$ and $x_{3}$, and the quantity of the estimated lumped disturbances is obtained as follows:

$$
\hat{a}(t)=-\mathrm{z}_{3}
$$

\subsection{Properties and Notations}

In the motion control system, the aim of the repetitive control is to track the position reference with the tracking error gradually convergent to zero after an amount of iterations.

The naturally properties for repetitive process are listed as follows:

Property 1: The position reference from the second iteration is designed as:

$$
\theta_{d}(t)=\theta_{d}\left(t-P_{t}\right), t \geq P_{t}
$$

where $\theta_{d}(t)$ is the position reference; $P_{t}$ represents the time period of one iteration. The time $t<P_{t}$ represents the first iteration, and means starting from the second iteration.

Property 2: The quantity of the lumped disturbances is limited as follows:

$$
|a(t)| \leq \eta
$$

where $\eta$ is a positive. And the difference of the lumped disturbances between two iterations is also restricted as below:

$$
a(t)=a\left(t-P_{t}\right)+a_{n}(t),\left|a_{n}(t)\right| \leq \xi, t \geq P_{t}
$$

where $a_{n}(t)$ represents the quantity of the aperiodic disturbances, $\xi \geq 0$.

Based on above properties, some special notations are listed for simplicity.

Notation 1:

$$
e_{\theta}(t)=\theta_{d}(t)-\theta(t)
$$

where $e_{\theta}(t)$ is the tracking error between the reference position and actual position.

Notation 2:

$$
S(t)=\lambda e_{\theta}+\dot{e}_{\theta}
$$

where $S(t)$ is the proportional-derivative form of $e_{\theta}(t)$; $\lambda$ is a positive gain.

Notation 3:

$$
e_{a}(t)=a(t)-\hat{a}(t)
$$

where $e_{a}(t)$ is the estimate error of the lumped disturbances.

\section{Periodic Adaptive Extended State Observer}

In this section, the detailed hybrid control structure with PAESO for repetitive motion control system of PMSM is proposed. Then, the stability of PAESO is proved.

\subsection{Design of Hybrid Controller}

As illustrated in 0 , the hybrid controller is composed of a 
feedback branch $C_{f b}$, a feedforward branch $C_{f f}$, and a PAESO $C_{p a}$. The PAESO is described by the normal ESO applied in the first iteration shown in 0 (a) and the refined ESO adopted from the second iteration given in 0 (b), respectively.
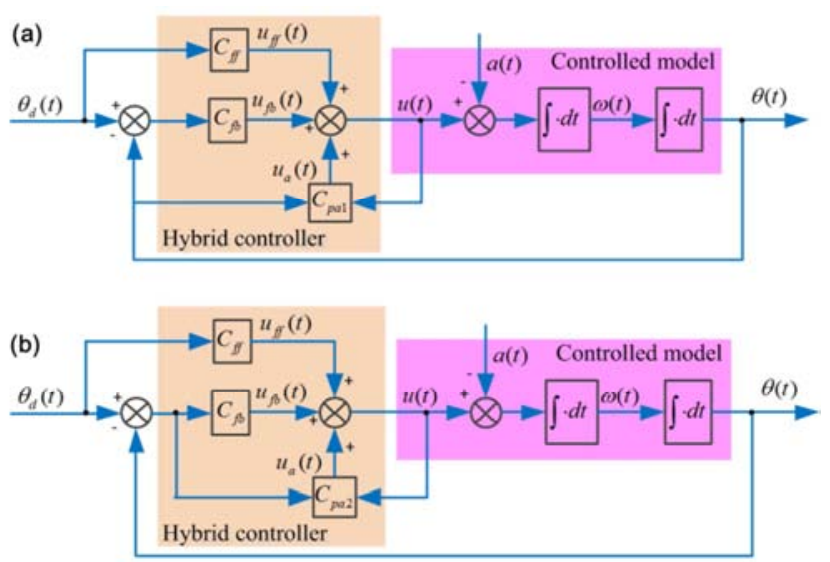

Figure 1. Structure of hybrid controller. (a) Applied in the first iteration, (b) Adopted from the second iteration.

Conventionally, the feedback branch $C_{f b}$ is designed for stability, and the feedforward branch $C_{f f}$ is developed to speed up the tracking response. The outputs of $C_{f b}$ and $C_{f f}$ are expressed as follows:

$$
\begin{gathered}
u_{f b}(t)=\alpha S(t)+\lambda \dot{e}_{\theta}(t)=(\alpha \lambda) e_{\theta}(t)+(\alpha+\lambda) \dot{e}_{\theta}(t) \\
u_{f f}(t)=\ddot{\theta}_{d}(t)
\end{gathered}
$$

where $\alpha$ is a positive gain.

The normal ESO $C_{p a 1}$, given in 0 (a), works in the first iteration for the purpose of capturing approximate range of lumped disturbances, and the estimated quantity of the lumped disturbances is stored in the memory as the initial value. The output of $C_{p a 1}$ is expressed below:

$$
u_{p a 1}(t)=\hat{a}(t)
$$

While starting from the second iteration, the refined ESO $C_{p a 2}$ presented in 0 (b) takes effect. Because of the phase hysteresis and amplitude distortion in the normal ESO, the estimated quantity of lumped disturbances obtained from the first iteration cannot describe actual disturbances exactly. Thus, starting from the second iteration, the quantity of stored lumped disturbances from previous iteration is updated and optimized by integrating the real-time position tracking error. The output of $C_{p a 2}$ is represented as follows:

$$
u_{p a 2}(t)=\hat{a}\left(t-P_{t}\right)+K S(t)
$$

where $K$ is a positive gain.

In conclusion, the total outputs of the hybrid controller are:

$$
u(t)=u_{f b}(t)+u_{f f}(t)+u_{p a}(t)
$$

where $u_{p a}(t)$ represents $u_{p a 1}(t)$ in the first iteration or $u_{p a 2}(t)$ from the second iteration.

(a)
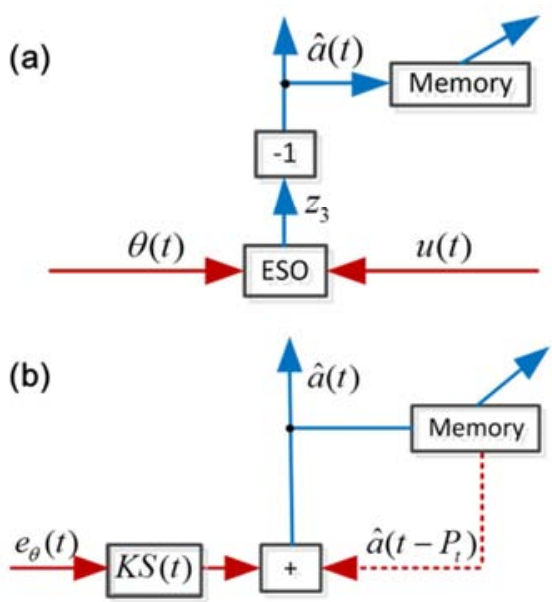

Figure 2. Structure of PAESO. (a) Normal ESO $C_{p a 1}$. (b) Refined ESO $C_{p a 2}$.

\subsection{Stability Analysis on Normal ESO}

In the first iteration, the output of the hybrid controller is:

$$
u(t)=u_{f b}(t)+u_{f f}(t)+u_{p a 1}(t)=\ddot{\theta}_{d}+\alpha S(t)+\lambda \dot{e}_{\theta}(t)+\hat{a}(t)
$$

Using (1) and (8), the following equation is obtained:

$$
\dot{S}(t)=e_{a}(t)-\alpha S(t)
$$

In order to prove the stability of the closed-loop system, (9) is reformed in $s$-domain:

$$
s S(s)+\alpha S(s)=e_{a}(s)
$$

Substituting (1) into (10), the overall closed-loop transfer function from the estimate error of the lumped disturbances to the position tracking error is obtained as follows:

$$
\frac{e_{\theta}(s)}{e_{a}(s)}=\frac{1}{(\mathrm{~s}+\lambda)(\mathrm{s}+\alpha)}
$$

Since all desired poles are located on the left-half plane, the system is stable [29].

\subsection{Stability Analysis on Refined ESO}

From the second iteration, the outputs of the hybrid controller are:

$$
\begin{aligned}
u(t) & =u_{f b}(t)+u_{f f}(t)+u_{p a 2}(t) \\
& =\ddot{\theta}_{d}+\alpha S(t)+\lambda \dot{e}_{\theta}(t)+\hat{a}\left(t-P_{t}\right)+K S(t)
\end{aligned}
$$

Using (10), (2) and (9), the following equation is obtained: 


$$
\dot{S}(t)+\alpha S(t)=e_{a}\left(t-P_{t}\right)+a_{n}(t)-K S(t)
$$

To prove the stability of the closed-loop system, consider the following positive Lyapunov function:

$$
V(t)=\frac{1}{2} S^{2}(t)+\frac{1}{2 K} \int_{t-P_{t}}^{t} e_{a}^{2}(\tau) d \tau
$$

Then, the difference of the positive Lyapunov function between two iterations is calculated as follows:

$$
\begin{aligned}
& \Delta V(t)=\frac{1}{2} S^{2}(t)-\frac{1}{2} S^{2}\left(t-P_{t}\right)+\frac{1}{2 K} \int_{t-P_{t}}^{t} e_{a}^{2}(\tau)-e_{a}^{2}\left(\tau-P_{t}\right) d \tau \\
& =\int_{t-P_{t}}^{t}-\left(\alpha+\frac{K}{2}\right) S^{2}(\tau)+\frac{1}{K} a_{n}(\tau) e_{a}\left(\tau-P_{t}\right)+\frac{1}{2 K} a_{n}^{2}(\tau) d \tau
\end{aligned}
$$

According to the condition in (9) and (10), (15) is rewritten as follows:

$$
\Delta V(t) \leq \int_{t-P_{t}}^{t}-\left(\alpha+\frac{K}{2}\right) S^{2}(\tau) d \tau+\frac{\gamma \xi P_{t}}{K}+\frac{\xi^{2} P_{t}}{2 K}
$$

where $\gamma=\int_{t-P_{t}}^{t} e_{a}\left(\tau-P_{t}\right) d \tau$ is a known integration from time instant $t-2 P_{t}$ to $t-P_{t}$.

If $K$ and $\alpha$ are appropriately selected, and the following condition is guaranteed:

$$
\int_{t-P_{t}}^{t}\left(\alpha+\frac{K}{2}\right) S^{2}(\tau) d \tau \geq \frac{\xi}{K} \gamma P_{t}+\frac{1}{2 K} \xi^{2} P_{t}
$$

Finally, the difference becomes negative semi-definite as follows:

$$
\Delta V(t) \leq 0
$$

Therefore, the system will be stable in spite of the aperiodic disturbances. In the idea case, there are no random disturbances in the lumped disturbances, i.e., $\xi=0$, (16) will be absolute negative semi-definite.

\section{Experimental Results}

In this section, real-time experiments are conducted on PMSM control system as shown in 0 to verify the effectiveness of PAESO. Three different S-shaped references with low-acceleration, medium-acceleration, and high-acceleration are chosen to evaluate the tracking performance of PAESO. The curves of position, speed, and acceleration in S-shaped reference are illustrated in 0 , respectively.

\subsection{Experimental Setup}

The experimental apparatus designed for the repetitive motion control of PMSM contains three major parts as depicted in Fig.3. The first part is a GK6061-6AC31 PMSM manufactured by Golden Age $\AA$, China. The specifications of the PMSM are listed in 0 , and the resolution of the built-in incremental encoder is 10000 pulses/rev. The second part is a bus-type commercial servo driver with a STM32 plus a FPGA, where STM32F103ZFT6 is mainly responsible for position and current control; while FPGA EP4CE22F17C8 accomplishes slave EtherCAT-bus protocols. The position control cycle is $0.2 \mathrm{~ms}$, and the current control cycle is $0.1 \mathrm{~ms}$. The third part is a CNC controller, which including an ARM-chip AM335X and a FPGA. A Linux ${ }^{\circledR}$ operation system with real-time kernel Xenomai ${ }^{\circledR}$ is embedded in $T I \AA$ AM3352. FPGA EP4C10F17C8N deals with the master EtherCAT-bus protocols. The bus communication cycle between $\mathrm{CNC}$ controller and servo driver is $1 \mathrm{~ms}$.

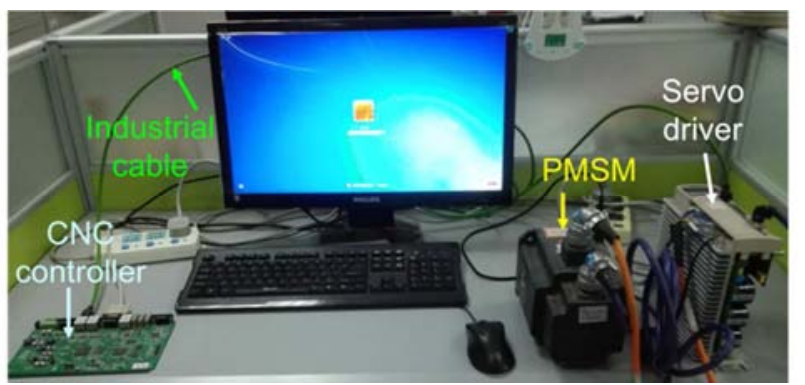

Figure 3. Experimental apparatus for repetitive position control of PMSM.

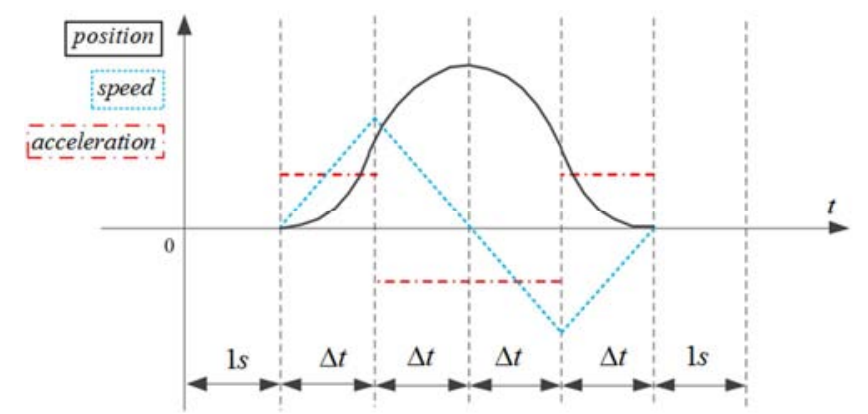

Figure 4. S-shaped reference. (a) acceleration (dashed red line). (b) speed (dotted blue line). (c) position (solid black line).

Table 1. Specifications of the PMSM.

\begin{tabular}{lll}
\hline Motor characteristics & Value & Units \\
\hline Rated current & 5.5 & $\mathrm{~A}$ \\
Rated speed & 2000 & $\mathrm{r} / \mathrm{min}$ \\
Rated torque & 6 & $\mathrm{~N} \cdot \mathrm{m}$ \\
Rotor inertia & 0.00087 & $\mathrm{Kg} \cdot \mathrm{m}^{2}$ \\
Torque coefficient & 1.09 & $\mathrm{~N} \cdot \mathrm{m} / \mathrm{A}$ \\
Mass & 10.6 & $\mathrm{Kg}$ \\
\hline
\end{tabular}

In the experiments, the hybrid controller is implemented in servo driver, while the experimental data, such as S-shaped reference with different accelerations and estimated quantity of lumped disturbances, are stored in the CNC controller. In the first iteration, $\mathrm{CNC}$ controller sends position reference to the servo driver, and receives the actual position and estimated quantity of the lumped disturbances from the servo driver simultaneously. From the second iteration, the CNC controller sends both the position reference and stored lumped disturbances to the servo driver, and receives the actual position and updated quantity of the lumped disturbances simultaneously.

The parameters of normal ESO in the experiments are 
$\beta_{1}=1000, \quad \beta_{2}=3000, \quad \beta_{3}=10000$ and $\delta=0.0002$ respectively.

\subsection{Evaluation of Tracking Performance}

To evaluate the tracking performance of PAESO, three experiments (Cases 1, 2, 3) with the S-shaped references under different accelerations are carried out.

Case 1: $\Delta t=0.500(\mathrm{~s}), a c c=4 \pi\left(\mathrm{rad} / \mathrm{s}^{2}\right)$;

Case 2: $\Delta t=0.250(\mathrm{~s}), a c c=16 \pi\left(\mathrm{rad} / \mathrm{s}^{2}\right)$;

Case 3: $\Delta t=0.125(\mathrm{~s}), a c c=64 \pi\left(\mathrm{rad} / \mathrm{s}^{2}\right)$.

where $\Delta t$ is the time spent in acceleration stage; acc is the acceleration of reference trajectory. The details of $\Delta t$ and $a c c$ are given in 0.

In Case 1, the parameters of the hybrid controller are chosen as follows: $\alpha=3, \lambda=100$, and $K=50$. 0 shows the tracking performance achieved by PAESO. As can be found from 0 (a) and (b) that at the first iteration, the tracking error is relatively large; from the second iteration, the tracking error converges towards zero asymptotically. It is clear that a better tracking performance is achieved due to the accurate compensation of the lumped disturbances, in other words, the PAESO gets more precise estimation of the lumped disturbances than the normal ESO. The quantity of the lumped disturbances obtained by PAESO and its FFT result are illustrated in 0 (c) and 0 (d), the harmonics exist besides not only $0 \mathrm{~Hz}$ but also $340 \mathrm{~Hz}$. It is implied that the lumped disturbances are well estimated and attenuated by PAESO since better tracking performance has been achieved.
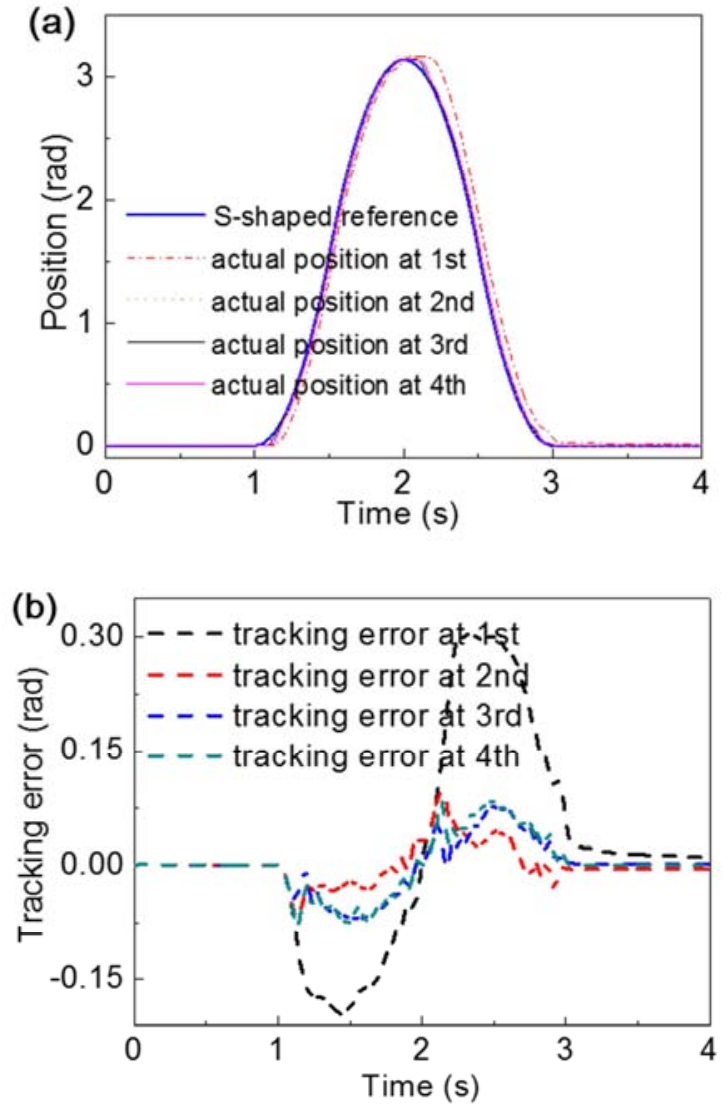
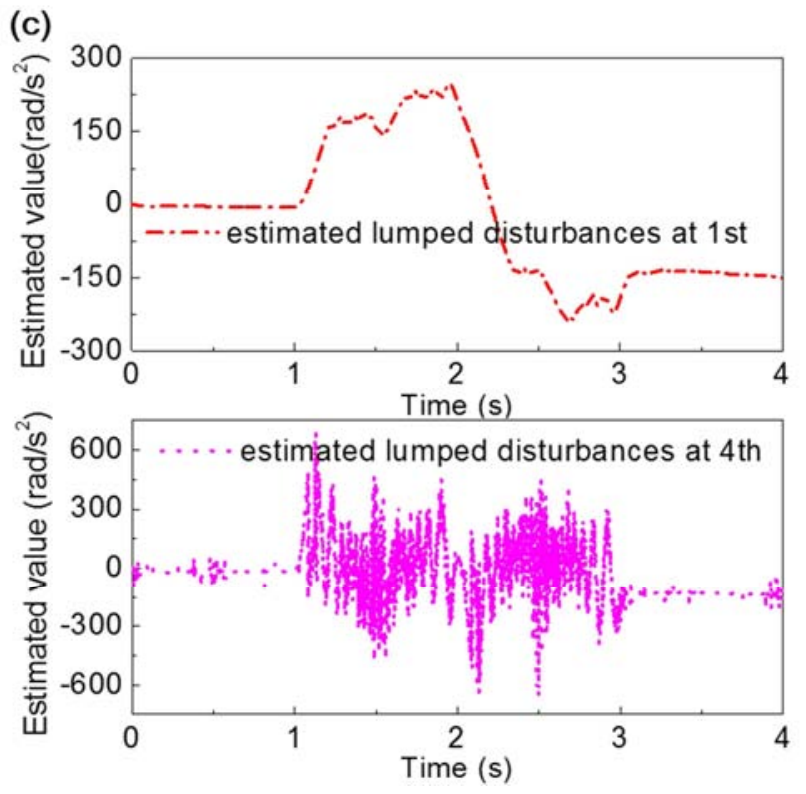

(d)
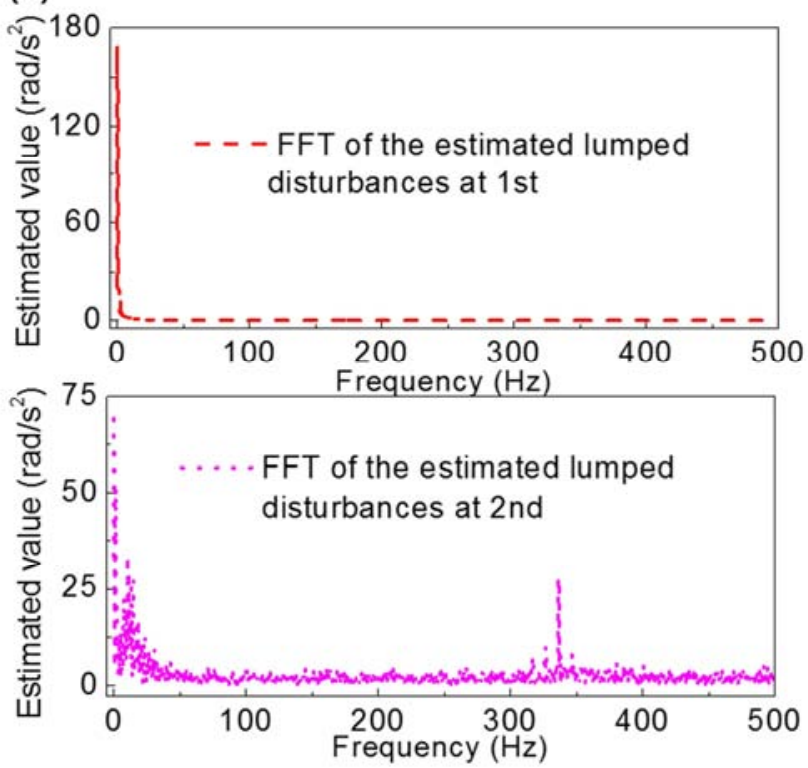

Figure 5. Tracking results in Case 1. (a) S-shaped reference and actual position. (b) position tracking error. (c) estimated lumped disturbances. (d) FFT results of estimated lumped disturbances.

In Case 2, $\alpha=3, \lambda=100, K=200$. The experimental result for Case 2 is given in 0 . As shown in 0 (a) and 0 (b), the tracking error achieved by PAESO from the second iteration is much smaller than that achieved by the normal ESO at the first iteration. As given in 0 (c) and (d), the PAESO improves the tracking performance by estimating much more harmonic parts of the lumped disturbances compared with the normal ESO. Therefore, it is obvious that PAESO has better approximation capacity of the lumped disturbances than the normal ESO. 

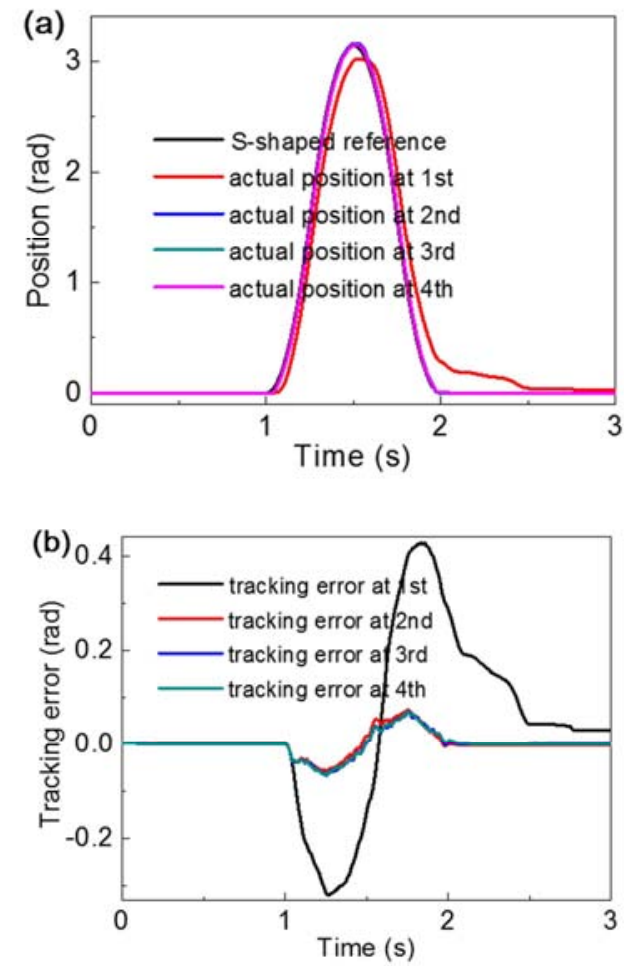

(c)
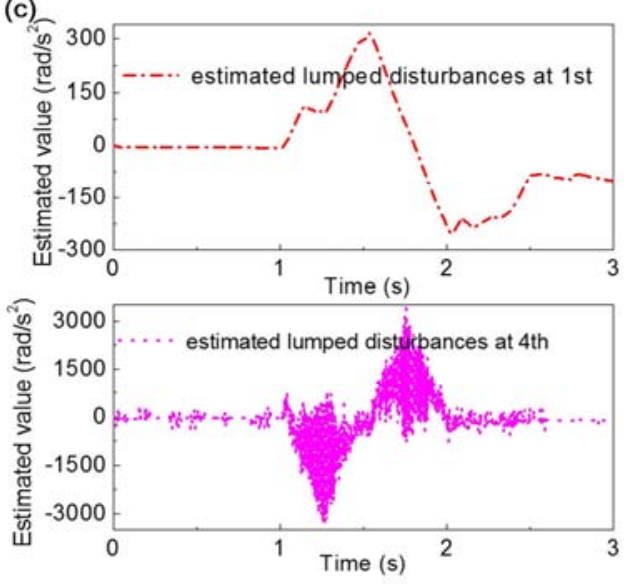

(d)
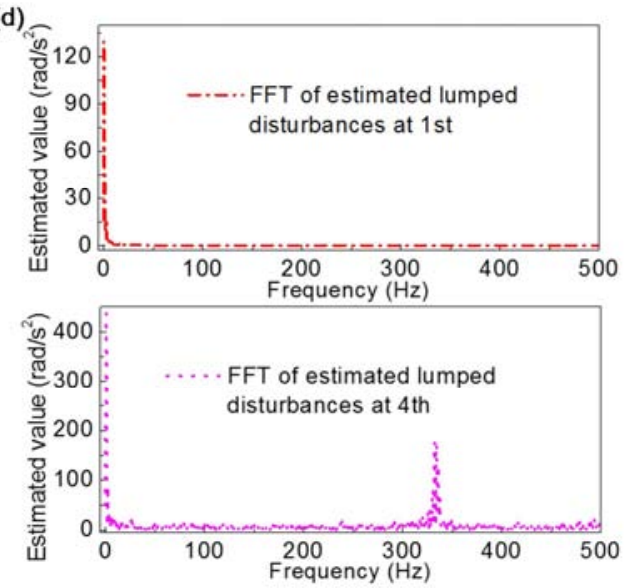

Figure 6. Tracking result in Case 2. (a) S-shaped reference and actual position. (b) position tracking error. (c) estimated lumped disturbances (d) FFT results of estimated lumped disturbances.
In Case 3, $\alpha=3, \lambda=100, K=800$. 0 illustrates the experimental results for Case 3 . The tracking performance obtained by normal ESO at first iteration is shown in 0 (a) and (b). Since only the harmonic part close to $0 \mathrm{~Hz}$ is estimated, tracking error is relatively large. However, from the second iteration, the tracking error is convergent since more harmonic part of the lumped disturbances is accurately estimated and rejected by PAESO as given in 0 (c) and (d).
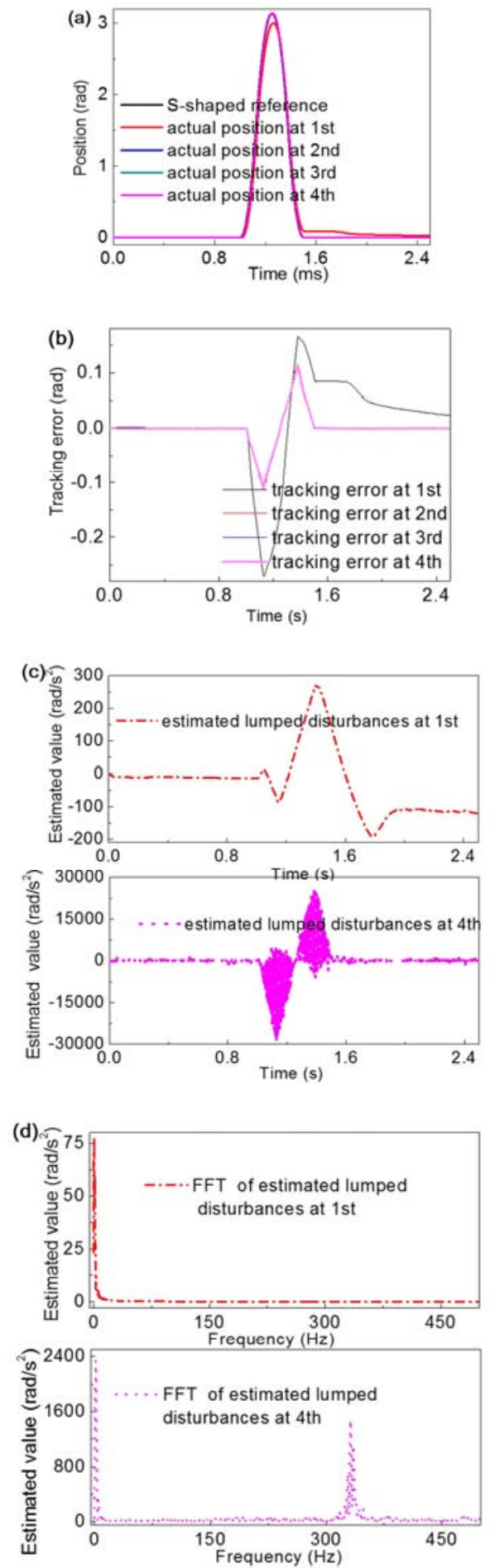

Figure 7. Tracking result in Case 3. (a) S-shaped reference and actual position. (b) position tracking error. (c) estimated lumped disturbances (d) FFT results of estimated lumped disturbances. 
From these results, it is verified that compared with the normal ESO, a better tracking performance can be achieved by PAESO by more accurate estimation of the lumped disturbances. It also can be found from 0 (d), 0 (d) and 0 (d) that, the harmonic frequencies at the forth iterations are almost the same in all FFT results, which confirms that the estimated results from PAESO is accurate and reliable.

\section{Conclusions}

This paper has presented a PAESO, consisting of normal ESO and refined ESO, for the estimation and compensation of lumped disturbances in the repetitive motion control system of PMSM. Firstly, a normal ESO has been developed to capture the approximated range of the lumped disturbances, and the estimated quantity is stored in the memory at the first iteration. Secondly, from the second iteration, a refined ESO has been established to update the estimated quantity of the lumped disturbances by integrating real-time position tracking error. Thirdly, it has been proved theoretically by the Lyapunov approach that the overall system is stable and the position tracking error will converge to zero asymptotically. Finally, three experiments has been conducted to demonstrate that the lumped disturbances are attenuated effectively by the proposed PAESO. Subsequently, it can be utilized in high performance repetitive motion control applications.

\section{Acknowledgements}

This work has been supported by the National Science Foundation of China (No. 11004055), the Fundamental Research Funds for the Central Universities (2016YQ11, 2016MS134, 2014MS162) and the Program for New Century Excellent Talents in University (NCET-12-0844).

\section{References}

[1] L. Hladowski, K. Galkowski, Z. Cai, E. Rogers, C. T. Freeman, and P. L. Lewin, "Experimentally supported 2D systems based iterative learning control law design for error convergence and performance," Control Engineering Practice, Vol. 18, 2010, pp. 339-348.

[2] M. F. Heertjes, and M. J. G. Ren, "Set-point variation in learning schemes with applications to wafer scanners," Control Engineering Practice, Vol. 17, 2009, pp. 345-356.

[3] K. K. Tan, T. H. Lee., H. F. Dou., S. J. Chin, and S. Zhao, "Precision motion control with disturbance observer for pulsewidth-modulated-driven permanent-magnet linear motors," IEEE Transactions on Magnetics, Vol. 39, 2003, pp. 1813-1818.

[4] S. Li, C. Xia, and X. Zhou, "Disturbance rejection control method for permanent magnet synchronous motor speed-regulation system," Mechatronics, Vol. 22, 2012, pp. 706-714.

[5] S. L. Chen, K. K. Tan, S. Huang, and C. K. Teo, "Modeling and compensation of ripples and friction in permanent-magnet linear motor using a hysteretic relay," IEEE/ASME
Transactions on Mechatronics, Vol. 15, 2010, pp. 586-594.

[6] H. M. Hasanien, "Torque ripple minimization of permanent magnet synchronous motor using digital observer controller," Energy Conversion and Management, Vol. 51, 2010, pp. 98-104.

[7] S. Chai, L. Wang, and E. Rogers, "A cascade MPC control structure for PMSM with speed ripple minimization," IEEE Transactions on Industrial Electronics, Vol. 60, 2013, pp. 2978-2987.

[8] W. H. Chen, J. Yang, L. Guo, and S. Li, "Disturbance observer-based control and related methods: An overview". IEEE Transactions on Industrial Electronics, 2015, pp.1083-1095.

[9] A.T. Elfizy, G. M. Bone, and M. A. Elbestawi, "Model-based controller design for machine tool direct feed drives," International Journal of Machine Tools and Manufacture, Vol. 44, 2004, pp. 465-477.

[10] Y. A. R. Mohamed, "Adaptive self-tuning speed control for permanent-magnet synchronous motor drive with dead time," IEEE Transactions on Energy Conversion, Vol. 21, 2006, pp. $855-862$.

[11] W. S. Huang, C. W. Liu, P. L. Hsu, and S. S. Yeh, "Precision control and compensation of servomotors and machine tools via the disturbance observer," IEEE Transactions on Industrial Electronics Vol. 57, 2010, pp. 420-429.

[12] C. Y. Chen, and M. Y. Cheng, "Adaptive disturbance compensation and load torque estimation for speed control of a servomechanism". International Journal of Machine Tools and Manufacture, Vol. 59, 2012, pp. 6-15

[13] H. Fujimoto, F. Kawakami, and S. Kondo, "Repetitive control of hard disk drives based on switching scheme: Experimental verification for multiple mode disturbance," The 8th IEEE International Workshop on Advanced Motion Control, 2004, pp. 323-328.

[14] J. Han, "From PID to active disturbance rejection control," IEEE Transactions on Electronics Vol. 56, 2009, pp. 900-906.

[15] S. Li, C. Xia, and X. Zhou, "Disturbance rejection control method for permanent magnet synchronous motor speed-regulation system," Mechatronics, Vol. 22, 2012, pp. 706-714.

[16] S. Li, and Z. Liu, "Adaptive speed control for permanent-magnet synchronous motor system with variations of load inertia," IEEE Transactions on Industrial Electronics, Vol. 56, 2009, pp. 3050-3059.

[17] G. Feng, Y. F. Liu, and L. Huang, "A new robust algorithm to improve the dynamic performance on the speed control of induction motor drive," IEEE Transactions on Power Electronics, Vol. 19, 2004, pp. 1614-1627.

[18] Y. X. Su, C, H. Zheng, and B. Y. Duan, "Automatic disturbances rejection controller for precise motion control of permanent-magnet synchronous motors," IEEE Transactions on Industrial Electronics, Vol. 52, 2005, pp. 814-823.

[19] D. Wu, and K. Chen, "Limit cycle analysis of active disturbance rejection control system with two nonlinearities," ISA transactions, Vol. 53, 2014, pp. 947-954. 
[20] W. Qian, S. K. Panda, and J.-X. Xu, "Torque ripple minimization in PM synchronous motors using iterative learning control," IEEE Transactions on Power Electronics, Vol. 19, 2004, pp. 272-279.

[21] J.-X. Xu, S. K. Panda, Y.-J. Pan, T. H. Lee, and B. H. Lam, “A modular control scheme for PMSM speed control with pulsating torque minimization," IEEE Transactions on Industrial Electronics, Vol. 51, 2004, pp. 526-536.

[22] P. Mattavelli, L. Tubiana, and M. Zigliotto, "Torque-ripple reduction in PM synchronous motor drives using repetitive current control," IEEE Transactions on Power Electronics, Vol. 20, 2005, pp. 1423-1431.

[23] H. S. Ahn, Y. Q. Chen, and H. Dou, "State-periodic adaptive compensation of cogging and coulomb friction in permanent magnet linear motors," American Control Conference, 2005, pp. 3036-3041.

[24] Y. Luo, Y. Q. Chen, and Y. G. Pi, "Cogging effect minimization in PMSM position servo system using dual high-order periodic adaptive learning compensation," ISA transactions, Vol. 49, 2010, pp.479-488.

[25] Y. Luo, Y. Q. Chen, H. S. Ahn, and Y. G. Pi, "Dynamic high order periodic adaptive learning compensator for cogging effect in permanent magnet synchronous motor servo system," IET Control Theory and Applications, Vol. 5, 2011, pp. 669-680.

[26] H. S. Ahn, Y. Q. Chen, and W. Yu, "Periodic adaptive compensation of state-dependent disturbance in a digital servo motor system," International Journal of Control Automation and Systems, Vol. 5, 2007, pp. 343-348.

[27] H. S. Ahn, and Y. Q. Chen, "State-dependent friction force compensation using periodic adaptive learning control," Mechatronics, Vol. 19, 2009, pp. 896-904.

[28] H. S. Ahn, and Y. Q. Chen, "State-dependent periodic adaptive disturbance compensation," IET Control Theory and Applications, Vol. 1, 2007, pp. 1008-1014Ahn. H. S., and Chen, Y. Q., "State-periodic adaptive friction compensation," The 16-th IFAC World Congress. Vol.38, 2005, pp.7-12.

[29] K. Cho, J. H. Kim, S. B. Choi, “A High-Precision Motion Control Based on a Periodic Adaptive Disturbance Observer in a PMLSM,"Vol. 20, 2015, pp. 2158-2171.

[30] K. Cho, H. Park, S. Choi, and S. Oh, "Precision motion control based on a periodic adaptive disturbance observer" 38th Annual Conference on IEEE Industrial Electronics Society, 2012, pp. 3832-3837. 